\title{
Fatores indicadores da sintomatologia climatérica
}

\author{
Predicting factors of climacteric symptoms
}

\author{
Dino Roberto Soares De Lorenzi ${ }^{1}$, Claudia Danelon², Bruno Saciloto ${ }^{3}$, Irineu Padilha Jr. ${ }^{4}$
}

\section{RESUMO}

Objetivo: identificar fatores indicadores da sintomatologia climatérica. Pacientes e Métodos: estudo transversal de 254 mulheres pós-menopáusicas com idade entre 45 e 60 anos atendidas em Ambulatório de Climatério entre junho e outubro de 2002. Foram excluídas mulheres histerectomizadas ou sob terapia hormonal. As atitudes sobre a menopausa foram avaliadas por meio de instrumento construído a partir da adaptação de um questionário, constituindo-se de 11 questões referentes a atitudes positivas (vantagens) e 11 referentes a atitudes negativas (desvantagens) sobre a menopausa. Para a coleta de dados sociodemográficos e relacionados a variáveis reprodutivas e às condições de saúde da população estudada, utilizouse questionário estruturado e previamente testado. A sintomatologia climatérica foi avaliada pelo índice de Kupperman e as atitudes sobre a menopausa por meio de instrumento específico. Os dados foram analisados pelo teste $t$ de Student, análise de variância (ANOVA) e regressão linear múltipla. Resultados: de modo geral, a sintomatologia climatérica foi leve em $28 \%$, moderada em $42,3 \%$ e intensa em $30,7 \%$ dos casos. Os sintomas mais prevalentes foram a irritabilidade $(87,1 \%)$, as artralgias/ mialgias $(77,5 \%)$ e a melancolia/tristeza $(73,2 \%)$, ao passo que os mais intensos foram as ondas de calor, a irritabilidade e a insônia. Sintomas vasomotores foram referidos por $60,2 \%$ das entrevistadas. Mostraram-se indicadores da sintomatologia climatérica a cor, a atividade física regular e as atitudes com respeito a menopausa. A cor branca $(p=0,02)$, a atividade física regular $(p=0,04)$ e uma percepção positiva sobre a menopausa $(p=0,01)$ associaram-se a sintomas climatéricos menos intensos. Em contrapartida, a percepção da menopausa como evento desvantajoso (atitude negativa) associou-se a pior sintomatologia climatérica $(\mathrm{p}<0,01)$. Conclusões: no presente estudo, a sintomatologia climatérica foi influenciada por fatores psicossociais e pela atividade física, além do estado de hipoestrogenismo característico desse período.

PALAVRAS-CHAVE: Climatério; Menopausa; Sintomas climatéricos; Índice de Kupperman

\section{ABSTRACT}

Purpose: to identify factors associated with climacteric symptoms. Methods: a cross-sectional study of 254 women aged between 45 and 60 years was carried out at the Climacterium Outpatient Clinic of Caxias do Sul University, RS, from June to October 2002. Women with previous hysterectomy or under hormonal therapy were excluded. The climacteric symptoms were evaluated by means of the Kuppermann index and the attitudes toward menopause by a specific questionnaire. Data were analyzed by Student's t test, analysis of variance (ANOVA) and multiple linear regression analysis. Results: twenty eight percent of the women reported mild climacteric symtoms, whereas $42.3 \%$ reported moderate symptoms and $30.7 \%$, intense symptoms. The most prevalent symptoms were: irritability $(87.1 \%)$, arthralgias/myalgias $(77.5 \%)$ and melancholy (73.2\%), while the most severe were hot flushes in $60.2 \%$ of the women, irritability and insomnia. Attitudes toward menopause, skin color and physical activity were predictors of climacteric symptoms. Positive attitudes toward menopause $(\mathrm{p}=0.01)$, white color $(\mathrm{p}=0.02)$ and the habit of practicing physical activity $(0.04)$ were associated with less intense climacteric symptoms. Negative attitudes toward menopause were associated with worse climacteric symptoms $(p<0.01)$. Conclusions: in the current study, the climacteric symptoms were influenced by psychosocial factors and physical activity, as well as by climacteric hypoestrogenism.

KEYWORDS: Climacterium; Menopause; Climacteric symptoms; Kupperman index

Unidade de Ensino em Tocoginecologia da Universidade de Caxias do Sul

1 Professor de Ginecologia da Universidade de Caxias do Sul

2 Médica Residente em Ginecologia do Hospital Geral

3,4 Acadêmico de Medicina da Universidade de Caxias do Sul

Correspondência: Dino Roberto Soares De Lorenzi

Rua Bento Gonçalves 1759/ 602 - 95020412 - Caxias do Sul - RS - e-mail: dlorenzi@terra.com.br

Recebido em: 10/9/2004 Aceito com modificações em: 15/12/2004 
Introdução

Como marco biológico, o climatério representa a transição entre a fase reprodutiva e a não reprodutiva, ou seja, do menacne para a senilidade, com conseqüências sistêmicas e potencialmente patológicas ${ }^{1}$. É fenômeno físiológico decorrente do esgotamento dos folículos ovarianos que ocorre em todas as mulheres de meia idade, seguido da queda progressiva da secreção de estradiol, culminando com a interrupção definitiva dos ciclos menstruais (menopausa) e o surgimento de sintomas característicos ${ }^{1,2}$.

Cerca de 60 a $80 \%$ das mulheres refere algum tipo de sintomatologia durante o climatério ${ }^{3}$, a sua maioria atribuída ao estado de hipoestrogenismo. Em particular, são comuns as queixas relacionadas a sintomas vasomotores, ressecamento vaginal, dispareunia e urgência miccional, estas últimas decorrentes de atrofia urogenital, com importante repercussão na esfera sexual e na qualidade de vida feminina ${ }^{1-3}$.

Dificuldades cognitivas, instabilidade emocional e humor depressivo, por sua vez, têm sido igualmente relacionados ao climatério ${ }^{4}$. Não obstante, persistem controvérsias se estes são decorrentes somente da carência estrogênica ou de fatores psicossociais, em especial a percepção de envelhecimento. Possivelmente, os fatores sócio-culturais e psicológicos atuariam influenciando a aceitação e modulação da resposta dos sintomas climatéricos ${ }^{2,4}$. No Brasil, entretanto, são ainda poucos os estudos a abordar essa questão.

Os sintomas vasomotores e a vaginite atrófica parecem ser as únicas queixas realmente decorrentes do hipoestrogenismo, sendo, assim, influenciados pelo estado menopausal, o que parece não ocorrer com os sintomas psicológicos. Corroborando com esta hipótese, pesquisa realizada com mulheres climatéricas residentes no municipio de Campinas revelou alta prevalência de sintomas psicológicos, cuja prevalência ou intensidade não se correlacionaram com o estado menopausal $^{5}$. O mesmo estudo revelou que, com exceção da atrofia urogenital e das ondas de calor, os sintomas de natureza somática, a irritabilidade e a maior labilidade emocional parecem ser influenciados principalmente por fatores psicossociais ${ }^{6}$.

Possivelmente, os sintomas climatéricos decorrem da interação entre a carência estrogênica e fatores culturais, sociodemográficos e psicológicos. Nesse sentido, têm se destacado os estudos que enfatizam a influência das atitudes ou crenças femininas em relação à menopausa na intensidade da sintomatologia climatérica ${ }^{7}$.
Em 1993, Groeneveld et al. ${ }^{8}$ demonstraram que atitudes negativas em relação à menopausa associavam-se a pior sintomatologia climatérica ${ }^{8}$. Posteriormente, Olofsson e Collins ${ }^{9}$, em estudo realizado na Suécia, observaram que, com exceção da sintomatologia vasomotora, as demais queixas climatéricas (distúrbios do humor, alteração da libido, déficit cognitivo, distúrbios do sono e dores articulares) estavam fortemente associados a fatores psicossociais, estilo de vida e, principalmente, com a percepção da mulher acerca do que representa a menopausa para a sua vida?

Assim, o presente estudo teve por objetivo identificar fatores indicadores da sintomatologia climatérica entre um grupo de mulheres residentes na região sul do Brasil.

\section{Pacientes e Métodos}

Estudo transversal, prospectivo, no qual foram incluídas todas as mulheres pós-menopáusicas atendidas no Ambulatório de Climatério da Universidade de Caxias do Sul (UCS) no período de $1^{\circ}$ de agosto de 2002 e 31 de outubro de 2002 .

O estado menopausal foi definido segundo a história menstrual no último ano. Considerou-se pós-menopáusica toda mulher cujo último fluxo menstrual espontâneo ocorreu há pelo menos 12 meses $^{10}$.

Foram consideradas elegiveis as pacientes entre 45 e 60 anos, não usuárias de terapia hormonal ou contraceptivos hormonais e sem histerectomia prévia ${ }^{11,12}$.

Foram excluidas as mulheres nas faixas etárias de 40 a 44 anos e 61 a 65 anos, visto estas representarem os periodos extremos do climatério, quando a sintomatologia climatérica é muitas vezes menos intensa ou mesmo ausente ${ }^{10-12}$. A exclusão das mulheres em terapia hormonal ou usuárias de contraceptivos hormonais até seis meses antes da sua possivel inclusão justifica-se por estas medicações interferirem na sintomatologia climatérica ${ }^{13,14}$. A impossibilidade de se estabelecer pela anamnese o seu estado menopausal implicou também a exclusão das mulheres com histerectomia prévia ${ }^{10-12}$.

A coleta de dados foi realizada mediante entrevistas realizadas por acadêmicos do curso de Medicina da UCS previamente treinados.

A sintomatologia climatérica foi avaliada por meio do índice de Kupperman (IK) ${ }^{13}$. Este é um dos istrumentos mais utilizados na avaliação clínica da sintomatologia climatérica, envolvendo onze 
sintomas ou queixas (sintomas vasomotores, insônia, parestesia, nervosismo, melancolia, vertigem, fraqueza, artralgia/mialgia, cefaléia, palpitação e zumbidos), aos quais são atribuídas diferentes pontuações segundo a sua intensidade e prevalência. Os escores totais são classificados em leves (valores até 19), moderados (entre 20 e 35) ou intensos (maior que 35). Assim, quanto maior a pontuação obtida, mais intensa a sintomatologia climatérica.

As respostas obtidas foram classificadas numa escala de 5 pontos ("concordo muito"; "concordo"; "nem concordo e nem discordo"; "discordo"; "discordo muito") ${ }^{8}$.

Foram assim pesquisados: idade, renda familiar per capita em salários mínimos (total dos rendimentos dos indivíduos economicamente ativos da família da entrevistada dividido pelo número de indivíduos que a integram), escolaridade (total de anos completos de estudo), cor (esta definida pela própria paciente como branca ou não branca), ocupação e estado marital (esta definida em com ou sem companheiro fixo) ${ }^{8,11,12}$.

As variáveis reprodutivas compreenderam o número referido de relações sexuais no último mês, o número de gestações e a idade de ocorrência da menopausa. As variáveis relacionadas às condições de saúde das mulheres pesquisadas referiram-se à confirmação de tabagismo no último ano, ao índice de massa corporal (IMC) e à história de atividade física regular. Considerou-se atividade física regular uma freqüência mínima de três vezes por semana e uma duração aproximada de 60 minutos $^{14}$. O IMC, por sua vez, foi avaliado segundo os critérios propostos por Kopelman $(1994)^{15}$, sendo considerada obesa toda mulher com IMC igual ou superior a $30 \mathrm{~kg} / \mathrm{m}^{2}$.

Os dados coletados foram analisados por meio do programa Statistical Package for Social Sciences for Personal Computer (SPSS-PC) versão 10. Inicialmente, procedeu-se à análise bivariada pelo coeficiente de correlação de Pearson, o teste $t$ de Student e a análise de variância (ANOVA). Para avaliação da associação entre as variáveis estudadas e o desfecho (sintomatologia climatérica segundo os escores do índice de Kupperman), procedeu-se à regressão linear múltipla (método Enter). Visando possibilitar a sua inclusão no modelo de regressão, as variáveis categóricas (tabagismo, estado marital, cor, ocupação e prática regular de exercícios físico) foram transformadas em variáveis dummy ${ }^{16}$. Em todas as análises realizadas, aceitou-se nivel de significância de $5 \%$.

O projeto foi previamente aprovado pelo Comitê de Ética em Pesquisa da Universidade de
Caxias do Sul. Antes da inclusão de qualquer paciente, esta foi esclarecida acerca da sua metodologia, objetivos e riscos, assinando após termo de consentimento livre e esclarecido.

\section{Resultados}

Foram entrevistadas 254 mulheres com média de idade de 54 anos $( \pm 4,1)$. A maioria $(56,3 \%)$ referiu menos de cinco anos completos de estudo, sendo 10,6\% analfabetas. Cerca de 29,9\% confirmaram ocupação remunerada, com renda familiar média de 1,3 salário mínimo per capita $( \pm 1,2)$.

A média de idade da menarca foi de 13,3 anos $( \pm 1,9)$, ao passo que a menopausa ocorreu em média aos 47,7 anos $( \pm 4,6)$. Atividade sexual foi referida por $59,8 \%$ das pacientes, sendo que $63 \%$ tinham companheiro fixo. Quatorze entrevistadas $(5,5 \%)$ confirmaram nunca ter engravidado.

Aproximadamente $29,1 \%$ das pacientes eram tabagistas. A prevalência de obesidade (IMC $\geq 30 \mathrm{~kg} / \mathrm{m}^{2}$ ) foi de $33,5 \%$, sendo que somente $27,6 \%$ $(\mathrm{n}=70)$ confirmaram atividade fisica regular.

A sintomatologia climatérica mostrou-se leve em $28 \%$, moderada em $41,3 \%$ e intensa em $30,7 \%$ dos casos. Os sintomas mais prevalentes foram: irritabilidade $(87,1 \%)$, artralgias / mialgias $(77,5 \%)$ e melancolia/tristeza $(73,2 \%)$. Os sintomas vasomotores foram referidos por $60,2 \%$ das pacientes (Tabela 1 ), sendo moderados e severos em $16,1 \%$ e $30,7 \%$ dos casos, respectivamente.

Tabela 1 - Prevalência dos diferentes sintomas climatéricos referidos pelas pacientes.

\begin{tabular}{lcc}
\hline Sintomas & $\mathbf{n}$ & $\%$ \\
\hline Irritabilidade & 221 & 87,1 \\
Artralgia/mialgia & 197 & 77,5 \\
Melancolia/tristeza & 186 & 73,2 \\
Zumbidos & 173 & 68,1 \\
Insônia & 169 & 66,5 \\
Parestesias & 164 & 64,5 \\
Cefaléia & 163 & 64,1 \\
Fogachos & 153 & 60,2 \\
Vertigem & 150 & 59,1 \\
Fraqueza/cansaço & 148 & 58,2 \\
\hline
\end{tabular}

Não se constatou qualquer associação entre a sintomatologia climatérica e a idade, a con- 
firmação de uma ocupação remunerada e o estado marital. Por sua vez, as mulheres com uma menor escolaridade, que não se consideraram de cor branca e com menor renda familiar per capita apresentaram sintomas climatéricos significativamente mais intensos (Tabela 2).

Tabela 2 - Associação entre a intensidade da sintomatologia climatérica (índice de Kupperman) e variáveis sociodemográficas.

\begin{tabular}{|c|c|c|c|}
\hline & n (\%) & Índice de Kupperman Média (DP) & $\mathrm{p}$ \\
\hline \multicolumn{4}{|l|}{ Idade (em anos) } \\
\hline $45-49$ & $44(17,3)$ & $27,9(13,7)$ & $0,31^{*}$ \\
\hline $50-54$ & $86(33,9)$ & $27,5(12,0)$ & \\
\hline $55-60$ & $124(48,8)$ & $25,2(13,2)$ & \\
\hline Média (DP) & $54(4,0)$ & & \\
\hline \multicolumn{4}{|l|}{ Cor } \\
\hline Branca & $178(70,1)$ & $24,9(12,6)$ & \\
\hline Não branca & $76(29,9)$ & $29,9(13,2)$ & $<0,01^{* \star}$ \\
\hline \multicolumn{4}{|l|}{ Anos completos de estudo } \\
\hline$<5$ & $143(56,3)$ & $28,5(13,1)$ & \\
\hline $5-9$ & $81(31,9)$ & $25,7(12,1)$ & $<0,01^{*}$ \\
\hline$\geq 10$ & $30(11,8)$ & $18,9(11,2)$ & \\
\hline Média (DP) & $4,8(3,6)$ & & \\
\hline \multicolumn{4}{|l|}{ Ocupação remunerada } \\
\hline Sim & $76(29,9)$ & $25,7(12,4)$ & \\
\hline Não & $178(70,1)$ & $28,2(13,9)$ & $0,18^{* *}$ \\
\hline \multicolumn{4}{|l|}{$\begin{array}{l}\text { Renda familiar per capita } \\
\text { em salários mínimos }\end{array}$} \\
\hline$<1$ & $143(56,3)$ & $28,2(12,8)$ & \\
\hline $1-2$ & $71(31,9)$ & $26,4(12,3)$ & $0,03^{*}$ \\
\hline$\geq 3$ & $30(11,8)$ & $20,4(12,7)$ & \\
\hline Média (DP) & $4,0(8,3)$ & & \\
\hline \multicolumn{4}{|l|}{ Estado marital } \\
\hline Com companheiro fixo & $160(63,0)$ & $26,8(12,4)$ & \\
\hline Sem companheiro fixo & $94(47,0)$ & $25,8(13,8)$ & $0,19^{* *}$ \\
\hline Total & $506(100)$ & & \\
\hline
\end{tabular}

*ANOVA / ** Teste $t$ de Student.

Teste de comparações múltiplas de Tukey:

Escolaridade: diferenças significativas $(p<0,05)$ foram observadas entre as mulheres com menos de 5 anos e os demais grupos avaliados. Não se evidenciaram diferenças significativas entre as mulheres com 5 a 9 anos e 10 ou mais anos completos de estudo.

Renda familiar: diferenças significativas $(p<0,05)$ foram observadas entre o grupo que recebia menos de um salário mínimo per capita e o grupo que recebia entre 1 e 2 salários mínimos per capita. 0 mesmo foi observado entre as mulheres com renda entre 1 e 2 salários e o grupo que recebia dois ou mais salários mínimos per capita.

A sintomatologia climatérica mostrou-se significativamente mais intensa entre as mulheres que não tinham uma atividade fisica regular. Em compensação, não se constatou associação significativa entre os sintomas climatéricos referidos com o hábito de fumar, o IMC, a idade da menopausa e o número de gestações.

A atividade sexual se correlacionou negativamente com a intensidade dos sintomas climatéricos. Assim, quanto maior o número de relações sexuais no último mês, menor a intensidade da sintomatologia climatérica (Tabela 3).

As atitudes em relação à menopausa correlacionaram-se significativamente com a sintomatologia climatérica. A percepção da menopausa como evento vantajoso (atitude positiva) associou-se a uma menor sintomatologia climatérica $(\mathrm{R}=-0,42 / \mathrm{p}<0,01)$, ao passo que as atitudes negativas sobre a menopausa associaram a sintomas mais intensos $(R=0,55 / p<0,01)$. 
Tabela 3 - Associação entre sintomatologia climatérica (escores do índice de Kupperman) e variáveis reprodutivas e relacionadas às condições de saúde da população estudada.

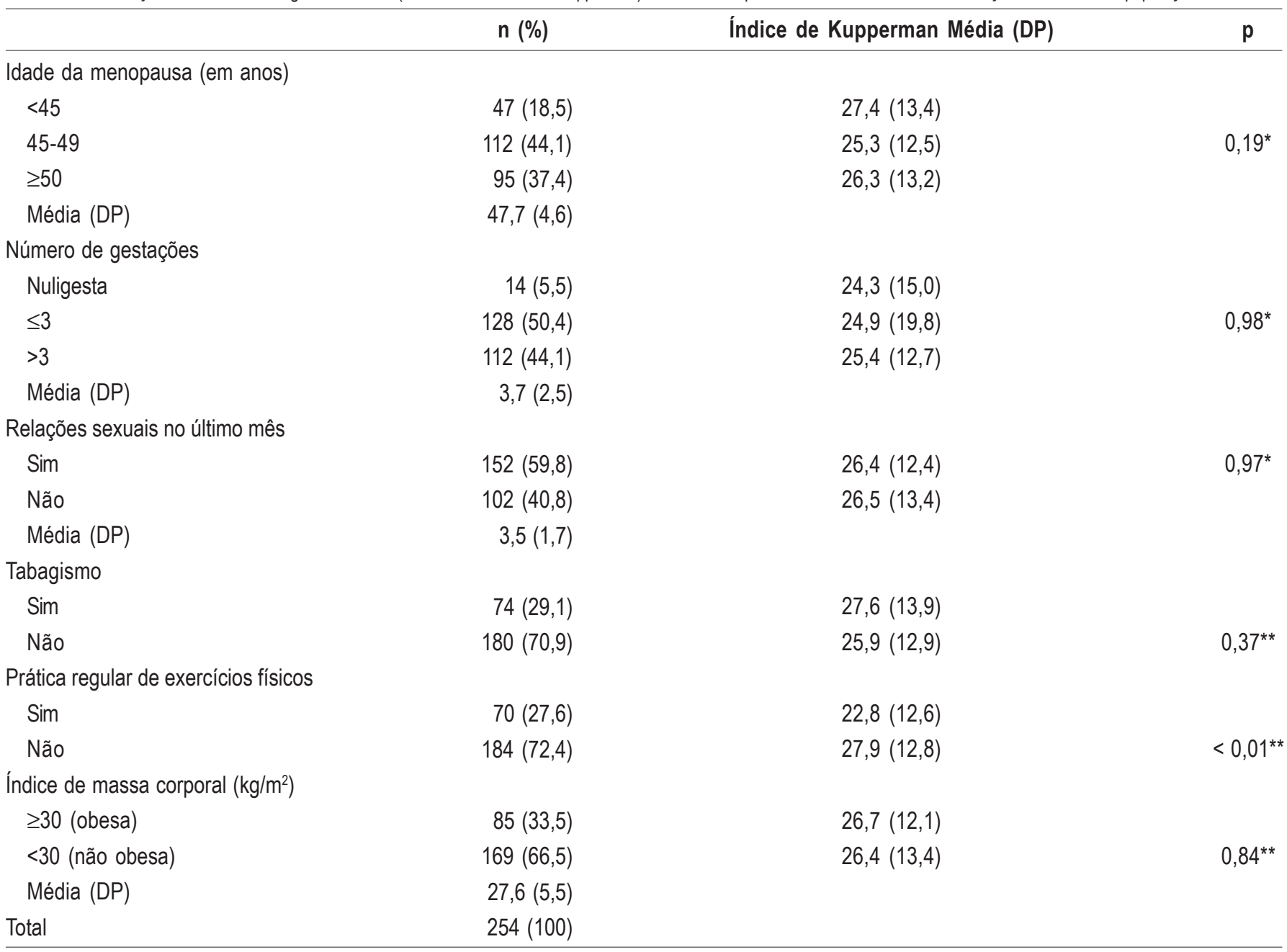

${ }^{*}$ ANOVA/**Teste $t$ de Student.

Em face da possibilidade da interferência de variáveis de confusão nas análises anteriores, procedeu-se à regressão linear múltipla, tendo como variável de desfecho a sintomatologia climatérica (indice de Kupperman). Confirmaram-se como indicadores da sintomatologia climatérica as atitudes em relação à menopausa, a cor e a prática regular de exercícios físicos. Assim, a cor branca, as atitudes positivas em relação à menopausa e a prática regular de exercícios físicos associaram-se a menor sintomatologia climatérica. Em contrapartida, entre as mulheres que não se consideraram de cor branca, sem atividade fisica regular e com atitudes negativas sobre a menopausa, os sintomas climatéricos revelaram-se significativamente mais intensos (Tabela 4).

\begin{tabular}{|c|c|c|}
\hline Variáveis* & $\beta$ & $\mathbf{P}$ \\
\hline Cor (branca) & $-0,125$ & 0,02 \\
\hline Prática regular de exercícios físicos & $-0,111$ & 0,04 \\
\hline Atitudes positivas frente à menopausa (vantagens) & $-0,155$ & 0,01 \\
\hline $\begin{array}{l}\text { Atitudes negativas em face da menopausa (des- } \\
\text { vantagens) }\end{array}$ & 0,348 & 0,01 \\
\hline
\end{tabular}

*Análise ajustada para: idade, escolaridade (anos completos de estudo), cor, ocupação remunerada, renda familiar per capita, estado marital, número de gestações, número de relações sexuais no último mês, história de tabagismo, prática regular de exercícios e índice de massa corporal.

\section{Discussão}

No presente estudo, a sintomatologia climatérica revelou-se moderada em $41,3 \%$ e intensa em $30,7 \%$ dos casos. As ondas de calor foram referidas por $60,2 \%$ das entrevistadas, 
percentual este próximo aos 55\% relatado entre as mulheres inglesas, porém inferior à prevalência descrita nos Estados Unidos $(78 \%)^{11,17}$.

A idade em nenhum momento das análises realizadas se mostrou fator indicador da sintomatologia climatérica, achado este semelhante ao obtido por outros autores ${ }^{18}$.

A maior prevalência de irritabilidade, melancolia ou tristeza entre as mulheres pesquisadas merece atenção. É necessário considerar que, ao procurarem assistência médica, as mulheres climatéricas tendem a atribuir à menopausa tanto os sintomas relacionados à carência estrogênica, como as queixas físicas ou emocionais decorrentes de estados mórbidos prévios, o que pode ter ocorrido entre a população estudada. Para Hunter ${ }^{12}$, é fundamental a diferenciação da verdadeira natureza dos sintomas referidos durante o climatério, em especial os relacionados à depressão.

A maior prevalência de estados depressivos no climatério estaria associada ao medo de envelhecer, sentimentos de inutilidade e até carência afetiva $^{4}$. O climatério coincide com o crescimento e independência dos filhos, viuvez e aposentadoria, eventos dificeis para mulher. Todavia, as mulheres com uma relação marital estável, profissionalmente realizadas e com atitudes positivas em relação ao envelhecimento tendem a referir menos sintomas depressivos. No presente estudo, porém, não se constatou associação entre o estado marital e a confirmação de uma ocupação remunerada com a intensidade da sintomatologia climatérica.

As queixas comuns de dificuldades com o sono entre as mulheres pesquisadas podem estar relacionadas à ocorrência de sintomas vasomotores e a estados de maior irritabilidade ${ }^{2}$. Mais recentemente, pesquisas têm revelado discordâncias entre a percepção da mulher climatérica quanto à qualidade do seu sono e os resultados de exames polissonográficos, achado este explicável pela maior prevalência de sintomas somáticos e psicológicos no climatério, o que poderia distorcer a sua percepção sobre o seu sono ${ }^{19}$.

Em contrapartida, os sintomas climatéricos mostram-se piores nas mulheres que não se consideraram de cor branca. A relação entre a cor e a intensidade da sintomatologia climatérica tem sido explorada por vários autores, todavia, seus achados são divergentes.

Em 2001, Avis et al. ${ }^{20}$ demonstraram que mulheres norte-americanas de etnia caucasiana tendem a apresentar mais queixas psicossomáticas, ao passo que as afro-americanas, mais sintomas vasomotores. Outros pesquisadores avaliaram a associação entre fatores demográficos e relacionados ao estilo de vida com a sintomatologia refe- rida por 16.065 mulheres climatéricas de diferentes grupos raciais. Em comparação com a raça caucasiana, as mulheres japonesas e chinesas apresentaram menos sintomas, ao passo que as afro-americanas, piores sintomas vasomotores, maior ressecamento vaginal e menos dificuldades com o sono. As mulheres hispânicas, por sua vez, referiram mais palpitações, perda urinária, secura vaginal e déficit da memória ${ }^{21}$.

Inicialmente, a escolaridade associou-se à sintomatologia climatérica, sendo esta menos intensa entre as mulheres com menos anos completos de estudo. Ainda que esse resultado não tenha se mantido por ocasião da análise multivariada, algumas considerações são importantes. Possivelmente, ao se aproximarem da menopausa, as mulheres trazem muitas dúvidas, o que pode gerar ansiedade e apreensão. Corroborando com isto, pesquisas demonstraram que as mulheres de meia idade submetidas a intervenções educativas expressam menos crenças negativas sobre a menopausa, com conseqüente redução dos níveis de ansiedade e menor sintomatologia climatérica ${ }^{22}$.

Mais da metade das entrevistadas confirmou atividade sexual no último ano, sendo que inicialmente a freqüência da atividade sexual associouse a menor sintomatologia climatérica. As dificuldades na esfera sexual seriam menores entre as mulheres que vivenciam positivamente o climatério. Estas percebem na redução das obrigações profissionais e com os filhos, uma oportunidade para um maior exercício afetivo-sexual. Entre as mulheres que associam a menopausa à velhice, por sua vez, a sexualidade pode vir a ser gravemente comprometida ${ }^{23}$.

Clinicamente, o hipoestrogenismo climatérico é responsável por atrofia urogenital, o que favorece a ocorrência de dispareunia. Paralelamente, o envelhecimento cutâneo por redução do colágeno e a tendência de acúmulo de gordura afetam a auto-imagem, contribuindo para menor auto-estima e até declínio do desejo sexual ${ }^{2,24}$. Os próprios sintomas vasomotores podem interferir na atividade sexual da mulher.

O medo de uma gestação indesejada, o constrangimento pelo desejo sexual e eventuais dificuldades no relacionamento com o parceiro são fatores que igualmente podem interferir na satisfação sexual feminina no climatério ${ }^{24}$.

Neste estudo, o tabagismo não se associou à sintomatologia climatérica. No entanto, pesquisas mostram que a nicotina interfere na globulina carreadora de estrogênio e acelera o processo de atresia folicular, antecipando a ocorrência da menopausa, além de agravar a sintomatologia 
climatérica ${ }^{25}$. A atividade fisica regular, por sua vez, associou-se à sintomatologia climatérica. Estudos indicam que as mulheres climatéricas que praticam regularmente exercícios físicos tendem a ter melhor humor, menor déficit de memória e menos sintomas somáticos ${ }^{26}$.

O exercício físico estimula a secreção de endorfinas hipotalâmicas, substâncias estas envolvidas na termorregulação hipotalâmica, reduzindo os sintomas vasomotores. Promove o fortalecimento muscular, a manutenção da mobilidade articular e da capacidade respiratória, além de menor acúmulo de gordura. A atividade física contribui ainda para a melhora da imagem corporal, aumentando a auto-estima feminina ${ }^{27}$.

O exercício físico regular tem sido recomendado na prevenção e tratamento dos processos de osteoartrite, estes, comuns no processo de envelhecimento, podendo ser causa de artralgias e até limitação dos movimentos ${ }^{26}$. É necessário lembrar que neste estudo, as queixas de artralgias e mialgias apresentaram elevada prevalência $(77,5 \%)$.

As atitudes ou crenças acerca da menopausa associaram-se significativamente à sintomatologia climatérica entre as mulheres entrevistadas, achado este análogo ao obtido por Groeneveld et al. ${ }^{8}$, cujas pesquisas mostraram que, entre as mulheres com percepção mais positiva da menopausa, os sintomas climatéricos eram menos intensos. Tal associação é explicável por possíveis mecanismos psicossomáticos ${ }^{8}$.

É reconhecido que as atitudes ou crenças femininas em relação à menopausa são fortemente influenciada por fatores culturais. Assim, nas sociedades orientais, nas quais a menopausa é fator de valorização feminino, visto o envelhecimento estar associado à sabedoria e experiência, os sintomas climatéricos tendem a ser menos intensos ou mesmo ausentes. No entanto, nas culturas ocidentais, em que a juventude feminina e a beleza são excessivamente valorizadas, a menopausa é freqüentemente percebida de forma negativa, estando associada ao envelhecimento e a maior proximidade da morte. Entre essas mulheres, a sintomatologia climatérica é freqüentemente mais intensa ${ }^{28}$.

Jiménez Lopez e Pérez Silva ${ }^{29}$, em estudo caso-controle, compararam a sintomatologia climatérica referida por mulheres com diferentes percepções e atitudes em relação à menopausa. A percepção da menopausa como evento negativo associou-se a maior prevalência de sintomas de natureza psicossocial.

Os dados obtidos a partir deste estudo, além do estímulo à atividade física regular, levam a salientar a importância da implementação de ativi- dades voltadas para a educação em saúde nas politicas de assistência à mulher climatérica. Estas, além de contribuírem para um maior autocuidado, contribuem para o esclarecimento de dúvidas, reduzindo o nivel de ansiedade e levando a uma percepção mais positiva acerca do climatério. Tal medida possivelmente se refletiria, inclusive, na adesão a eventuais tratamentos instituídos e na intensidade da sintomatologia climatérica.

Assim, ainda que não possam ser extrapolados para a totalidade da população feminina do Brasil, espera-se que os resultados deste estudo estimulem o desenvolvimento de futuras pesquisas sobre o impacto da síndrome climatérica na qualidade de vida feminina colaborando, se possivel, com o desenvolvimento de futuras políticas de saúde voltadas para a assistência à saúde da mulher brasileira no seu processo de envelhecimento.

\section{Referências}

1. Bossemeyer R. Aspectos gerais do climatério. In: Fernandes CE, Melo NR, Wehba S, editores. Climatério Feminino: fisiopatologia, diagnóstico e tratamento. São Paulo: Lemos Editorial; 1999. p. 17-33.

2. Aldrighi JM, Aldrighi CMS, Aldrighi APS. Alterações sistêmicas do climatério. Rev Bras Med. 2002; 59:15-21.

3. Speroff L, Glass RH, Kase NG. Clinical gynecologic endocrinology and infertility. 4th ed. Baltimore: Williams \& Wilkins; 1989. p. 134-55.

4. Dennerstein L, Lehert P, Guthrie J. The effects of the menopausal transition and biopsychosocial factors on well-being. Arch Women Ment Health. 2002; 5:15-22.

5. Pedro AO, Pinto-Neto AM, Costa-Paiva L, Osis MJ, Hardy E. Procura de serviço medico por mulheres climatéricas brasileiras. Rev Saúde Pública. 2001; 36:484-90.

6. Holte A. Influences of natural menopause on health complaints: a prospective study of healthy Norwegian women. Maturitas. 1992; 14:127-4.

7. Malacara JM, Canto de Cetina T, Bassol S, González N, Cacique L, Vera-Ramírez ML, Nava LE. Symptoms at pre- and postmenopause in rural and urban women from three States of Mexico. Maturitas. 2002; 43:11-9.

8. Groeneveld FP, Bareman FP, Barentsen R, Dokter HJ, Drogendijk AC, Hoes AW. Relationships between attitude towards menopause, well-being and medical attention among women aged 45-60 years. Maturitas. 1993; 17:77-88. 
9. Olofsson AS, Collins A. Psychosocial factors, attitude to menopause and symptoms in Swedish perimenopausal women. Climacteric. 2000; 3:33-42.

10.Rodrigues de Lima JG, Baracat EC. Sindrome do climatério. In: Rodrigues de Lima JG, Baracat EC, editores. Ginecologia Endócrina. São Paulo: Atheneu; 1995. p. 253-98.

11.Hunter M. The south-east England longitudinal study of the climacteric and postmenopause. Maturitas. 1992; 14:117-26.

12.Hunter MS. Predictors of menopausal symptoms: psychosocial aspects. Baillieres Clin Endocrinol Metab. 1993; 7:33-45.

13.Kupperman HS, Blatt MH, Wiesbader H, Filler W. Comparative clinical evaluation of estrogenic preparations by the menopausal and amenorrheal indices. J Clin Endocrinol Metab. 1953; 13:688-703.

14.Thompson PD, Buchner D, Pina IL, et al. Exercise and physical activity in the prevention and treatment of atherosclerotic cardiovascular disease: a statement from the Council on Clinical Cardiology (Subcommittee on Exercise, Rehabilitation, and Prevention) and the Council on Nutrition, Physical Activity, and Metabolism (Subcommittee on Physical Activity). Circulation. 2003; 107:3109-16.

15.Kopelman PG. Investigation of obesity. Clin Endocrinol (Oxf). 1994; 41:703-8.

16.Zar JH. Biostatistical analysis. $1^{\text {st }}$ ed. New Jersey: Prentice-Hall; 1984. p. 328-33.

17.von Mulhen DG, Kritz-Silverstein D, Barrett-Connor E. A community-based study of menopause symptoms and estrogen replacement in older women. Maturitas. 1995; 22:71-8.

18.Blummel JEM, Binfa LE, Gramegna GMS, Tacla XF, Aracena BP, Cumsille MAG. Efecto del climaterio en la calidad de vida. Rev Chil Obstet Ginecol. 1998; 63:437-45.

19.Polo-Kantola P, Erkkola R, Irjala K, Helenius H, Pullinen, Polo O. Climacteric symptoms and sleep quality. Obstet Gynecol. 1998; 94:219-24.
20.Avis NE, Stellato R, Crawford S, et al. Is there a menopausal syndrome? Menopausal status and symptoms across racial/ethnic groups. Soc Sci Med. 2001; 52:345-56.

21.Gold EB, Sternfeld B, Kelsey JL, et al. Relation of demographic and lifestyle factors to symptoms in a multi-racial/ethnic population of women 40-55 years of age. Am J Epidemiol. 2000; 152:463-73.

22.Liao KKM, Hunter MS. Preparação para a menopausa: avaliação das expectativas de uma intervenção educativa de saúde na mulher de meiaidade. Maturitas [edição brasileira]. 1999; 1:59-67.

23.Favarato MECS, Aldrighi JM, Fráguas Júnior R, Pires ALR, Lima SMRR. Sexualidade e climatério: influência de fatores biológicos, psicológicos e sócioculturais. Reprod Clim. 2000; 15:199-202.

24. Chiechi LM, Granieri M, Lobascio A, Ferreri R, Loizzi P. Sexuality in the climacterium. Clin Exp Obstet Gynecol. 1997; 24:158-9.

25.Nusbaum ML, Gordon M, Nusbaum D, McCarthy MA, Vasilakis D. Smoke alarm: a review of the clinical impact of smoking on women. Prim Care Update Ob Gyns. 2000; 7:207-14.

26. Slaven L, Lee C. Mood and symptom reporting among middle-aged women: the relationship between menopausal status, hormone replacement therapy and exercise participation. Health Psychol. 1997; 16:203-8.

27.Stevenson ET, Davy KP, Jones PP, Desouza CA, Seals DR. Blood pressure risks factors in healthy postmenopausal women: physical activity and hormone replacement. J Appl Physiol. 1997; 82:652-60.

28.Lolas Stepke F. Las ciencias sociales como discurso de la salud reproductiva. El ejemplo del climaterio femenino. Cad Saúde Pública. 1998; 14 (Supl 1):131-4.

29.Jimenez Lopez J, Pérez Silva G. Actitud de la mujer en la menopausia y su influencia en el climaterio. Ginecol Obstet Mex. 1999; 67:319-22. 